\title{
Antientzündliches Essen - Rheumaernährung konkret und praktisch
}

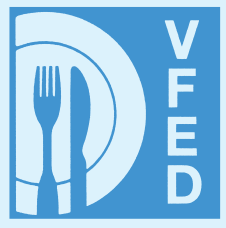

\author{
Birgit Blumenschein*
}

\begin{abstract}
„Es gibt keine Rheuma-Diät“ - das bekommen Patienten leider häufig zu hören und zu lesen. Ernährungsfachkräfte werden jedoch nicht müde zu erläutern, dass bestimmte Ernährungsmaßnahmen über die allseits bekannten DGE-Empfehlungen hinaus wichtig sind. Hier ist noch viel Aufklärungsarbeit für Rheumatiker notwendig.
\end{abstract}

Betroffene wollen wissen, wie aufwändig die Ernährung sein muss. Sie lesen und hören von Vegetarismus bis hin zur veganen Kost und dass sie auf Alkohol und Nikotin verzichten sollen. Irritierend sind die Empfehlungen, tierische Fette und v.a. Schweineschmalz zu meiden, überhaupt das Schweinefleisch wegzulassen und dafür Fisch zu essen. In Internetforen, in denen sich Rheumakranke austauschen, finden sich Befragungen, wer sich wie ernährt. Ergebnisse sind, dass zwei Drittel der Betroffenen weiterhin Fleisch essen, die meisten lediglich Produkte aus und vom Schwein weglassen. Die folgenden Ausführungen zeigen, dass nicht nur Schweinefleisch gemieden werden sollte.

\section{Vegetarier haben seltener Rheuma}

Sicher und über Untersuchungen nachgewiesen ist, dass Rheuma nur in Ausnahmefällen direkt durch die Ernährung ausgelöst wird. Als Ausnahmefälle werden Kollagenosen und dabei besonders der Lupus erythematodes und die bei diesen Menschen auftretenden Nahrungsmittelunverträglichkeiten (z.B. ge-

\footnotetext{
* Nach einem Vortrag anlässlich der 18. Aachener Diätetik Fortbildung des VFED e.V., 10.-12.9.2010
}

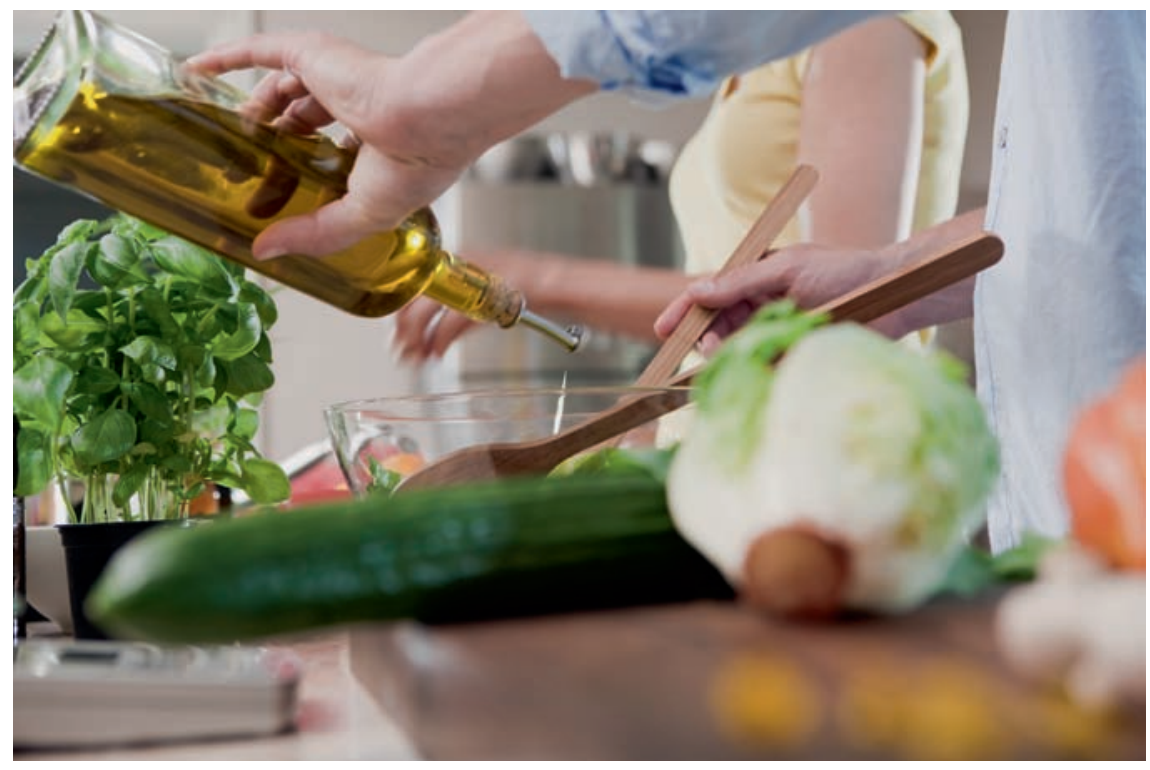

Selbst handeln: Mit ausgewählten Speisefetten - viel Omega-3, wenig Omega-6 - kann das Entzündungsgeschehen positiv beeinflusst werden. ๑ Westend61

genüber Laktose, Fruktose sowie Gluten) diskutiert. Nach aktueller Studienlage zeigt sich, dass Vegetarier tatsächlich deutlich weniger entzündlich-rheumatische Erkrankungen aufweisen als Mischköstler. Diese verzehren viele Lebensmittel, z. B. Fleisch und Fleischwaren, die entzündungsfördernde Substanzen enthalten.

Als Ursache für entzündlich-rheumatische Reaktionen wurden Prostaglandine erkannt und benannt. Diese entstehen aus der Omega-6-Fettsäure Arachidonsäure, die sowohl im Körper synthetisiert wird, als auch überwiegend in tierischen Lebensmitteln enthalten ist. Über die Nahrung zugeführte Arachidonsäure wird zu $90 \%$ in die Zellen resorbiert und steht dort zur Bildung der entzündungsfördernden Substanzen zur Verfügung. Als den Rheumaschub auslösend werden von Betroffenen verschiedene Lebensmittel genannt ( $\triangleright$ Tab. 1$)$.

\section{Erkenntnisse nutzen}

Der Verzehr von tierischen Fetten, besonders der Fette von rotem Fleisch, wird als für den Rheumatiker „schubunterhaltend“ bzw. „symptomverstärkend“ beschrieben. Parallel dazu scheinen $\mathrm{zu}$ wenig Fisch und zu geringe Mengen an Obst und Gemüse im täglichen Verzehr entzündungsunterhaltend zu wirken.

Antientzündliches Essen bedeutet nach heutigen Erkenntnissen

- das Erkennen und Meiden der krankheitsverstärkenden, entzündungsfördernden Lebensmittel (-inhaltsstoffe) und

- eine erhöhte Zufuhr von entzündungshemmenden, das Immunsystem stärkenden Lebensmittel (-inhaltsstoffen).

Eine praktische Umsetzung erfahren diese Überlegungen durch folgende Maßnahmen: 
- Tab. 1 Schubauslösende Lebensmittel bei rheumatoider Arthritis (in \% der Patientenangaben).

\begin{tabular}{|l|l|l|l|}
\hline Schwein & $39 \%$ & Hafer & $37 \%$ \\
\hline Rind & $32 \%$ & Eier & $32 \%$ \\
\hline Lamm & $17 \%$ & Roggen & $32 \%$ \\
\hline Mais & $57 \%$ & Kaffee & $32 \%$ \\
\hline Weizen & $54 \%$ & Erdnüsse & $20 \%$ \\
\hline Milch & $37 \%$ & Soja & $17 \%$ \\
\hline
\end{tabular}

Quelle: O. Adam: Ernährung bei rheumatischen Erkrankungen, ErnährungsUmschau 12/08, S. $734 \mathrm{ff}$.

- arachidonsäurearme Kost,

- Kost, reich an mehrfach ungesättigten Fettsäuren und

- Kost, reich an Vitamin E, Vitamin C, Selen und Zink.

Zur Osteoporoseprophylaxe (Osteoporose als eine mögliche Folge des Medikamenteneinsatzes bei Rheumatikern) wird flankierend kalziumreiches sowie phosphatarmes Essen und Trinken empfohlen. Aufgrund des Basistherapeutikums Methotrexat wird nach Absprache des Arztes oft eine Folsäuresupplementierung empfohlen.

\section{Arachidonsäure reduzieren}

Arachidonsäurearme Kost bedeutet, die Zufuhr an Arachidonsäure auf maximal $350 \mathrm{mg}$ pro Woche bzw. $50 \mathrm{mg}$ pro Tag zu begrenzen. Konkret und praktisch umgesetzt heißt das:

- 2 Fleischmahlzeiten pro Woche à $100 \mathrm{~g}$

- keine Innereien und daraus hergestellte Produkte

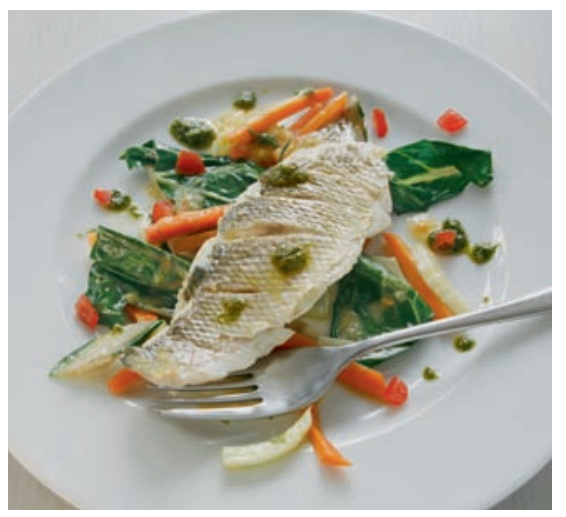

Fisch muss. Das gilt für Rheumatiker ganz besonders. (C) TVG/Chris Meier
- max. 2 Eigelb pro Woche

- eine Wurst-/Schinkenmahlzeit pro Woche

- nur fettarme Milch und Milchprodukte

- kein Schweineschmalz und/oder Rindertalg.

Die Einschränkung von Fleisch- und Wurstmahlzeiten bezieht sich auf alle Fleischarten. Es wird oft Geflügel statt Schweinefleisch empfohlen, doch auch Pute und Huhn enthalten ca. 160 bzw. über $200 \mathrm{mg}$ Arachidonsäure/100 g. Eine Ausnahme bildet Truthahnbrust ohne Haut mit 55 mg/100 g, bei der Keule ohne Haut sind es schon wieder $170 \mathrm{mg}$.

\section{Kaltwasserfische}

Insgesamt wird zu einer überwiegend (ovo-) lakto-vegetabilen Kost geraten, die mit entzündungshemmenden „Fischfetten“ aus Kaltwasserfischen wie Makrele, Hering, Lachs und Thunfisch ergänzt werden soll. Diese Empfehlung wird aufgrund darin enthaltenen Omega-3-Fettsäuren EPA (Eicosapentaensäure) und DHA (Docosahexaensäure) gegeben. Die erhöhte Zufuhr von Omega-3-Fettsäurereichen Pflanzenölen, wie z.B. Lein- und Rapsöl, wirkt synergistisch.

\section{Hürden bei der Umsetzung}

Allerdings wird offen über die Realisierbarkeit einer derart gemüse- und getreidelastigen (vegetabilen) Ernährung diskutiert. Problematisch ist sie für die Betroffenen häufig deshalb, weil Gemüse und Getreideprodukte zur wirklich gesunden Versorgung mit wertvollen Inhaltsstoffen frisch eingekauft und verzehrt werden sollten. Das Heben und Tragen der Einkaufstaschen, die häufigeren Einkaufstouren sowie kräftiges Kauen fordert von den Rheumakranken schlicht, ihre Schmerzen zu ignorieren. Hier scheitern viele gut gemeinte und ernährungsphysiologisch korrekte, aber eben nur theoretische Ernährungsempfehlungen an ihrer realistischen und praktischen Umsetzung.

Zusätzlich zur Empfehlung, wenig Arachidonsäure (AA) über die Lebensmittel aufzunehmen, kann mithilfe von mehrfach ungesättigten Fettsäuren die körpereigene Synthese der AA gehemmt werden. Das Enzym „Desaturase“, das die Umwandlung zu Arachidonsäure im Körper katalysiert, wird durch Linolsäure, $\alpha$-Linolensäure, EPA und DHA in seiner Wirkung gehemmt. Die alleinige Zufuhr der essenziellen Linolsäure reicht jedoch nicht aus, man müsste ca. $45 \mathrm{ml}$ Rapsöl oder gar $116 \mathrm{ml}$ Olivenöl pro Tag aufnehmen, um eine merkliche Enzymhemmung zu erreichen. Effektiver können hier die Öle mit Omega-3-Fettsäuren eingesetzt werden, die zusätzlich eine entzündungshemmende Wirkung entfalten - v.a. EPA. Es ist jedoch nicht möglich, diese Fettsäure über herkömmliche Lebensmittel isoliert aufzunehmen. Für Rheumatiker wird die tägliche Zufuhr von ca. 900-1000 mg EPA empfohlen (zum Vergleich für Gesunde lt. DGE: ca. 300 mg EPA/Tag).

Konkret und praktisch umgesetzt bedeutet das in diesem Fall:

- 70-85 g Hering/Tag oder

- $70 \mathrm{~g}$ Thunfisch/Tag oder

- 100 g Makrele/Tag oder

- 160 g Sardine/Tag oder

- 200 g Lachs/Tag oder

- 650 g Forelle/Tag !!! oder

- $1 \mathrm{~kg}$ Seelachs /Tag !!!

Dosenfisch enthält (wesentlich) weniger Omega-3-Fettsäuren als frischer Fisch ( Tab. 2).

\section{Geeignete Speisefette wählen}

Um die positiven Effekte der entzündungshemmenden Fettsäuren für den Körper optimal zu entfalten, sollte ein

- Tab. 2 Vergleich von Frischfisch und Dosenfisch.

\begin{tabular}{|l|l|}
\hline Beispiel & $\begin{array}{l}\text { Omega-3-FS- } \\
\text { Gehalt pro } \mathbf{1 0 0} \mathbf{~ g}\end{array}$ \\
\hline Sardinen, frisch & $1800 \mathrm{mg}$ \\
\hline Sardinen, Dose & $1000 \mathrm{mg}$ \\
\hline Thunfisch, frisch & $1500-2500 \mathrm{mg}$ \\
\hline $\begin{array}{l}\text { Thunfisch, } \\
\text { eingelegt }\end{array}$ & $300-700 \mathrm{mg}$ \\
\hline Hering, frisch & $1000-2000 \mathrm{mg}$ \\
\hline Bismarckhering & $900 \mathrm{mg}$ \\
\hline
\end{tabular}

Quellen: USDA Nutrient Database for Standard Reference, 2003; Souci, Fachmann, Kraut, 7. Aufl. 2008 
> Tab.3 Verhältnis von Omega-3-zu Omega-6Fettsäuren.

\begin{tabular}{l|l|l}
$\begin{array}{l}\text { SOLL } \\
\text { bei Rheuma }\end{array}$ & Omega-3 $:$ Omega-6 \\
& $<1: 4$ \\
\hline IST & \\
\hline Leinöl & $1: 0,2-0,4$ \\
\hline Perillaöl & $1: 0,25$ \\
\hline Rapsöl & $1: 2$ \\
\hline Walnussöl & $1: 4,5$ \\
\hline Sojaöl & $1: 7$ \\
\hline Weizenkeimöl & $1: 7$ \\
\hline Olivenöl & $1: 9$ \\
\hline Standardmargarine & $1: 10$ \\
\hline Palmöl & $1: 10$ \\
\hline Erdnüsse & $1: 26$ \\
\hline Sesam & $1: 27$ \\
\hline Maiskeimöl & $1: 61$ \\
\hline Sonnenblumenöl & $1: 126$ \\
\hline Distelöl & $1: 150$ \\
\hline
\end{tabular}

Quellen: Ernährung \& Medizin 2008; 23: 29-33, Ernährung \& Medizin 2010; 25 (Suppl. 1): 3-18

günstiges Verhältnis von Omega-3- zu Omega-6-Fettsäuren bevorzugt werden. Als wünschenswert für Rheumatiker wird ein Verhältnis von $1: 4$ angesehen. Im Bereich der Öle für die Zubereitung von kalten und/oder warmen Speisen entsprechen in ihrer Zusammensetzung

\begin{tabular}{|c|c|c|c|}
\hline Vitamin E & 100-200 mg/Tag & z. B. enthalten in & $\begin{array}{l}70 \mathrm{ml} \text { Weizenkeimöl } \\
230 \mathrm{ml} \text { Rapsöl } \\
600 \mathrm{~g} \text { Mandeln, süß } \\
2,5 \mathrm{~kg} \text { Walnüssen }\end{array}$ \\
\hline Vitamin C & $200 \mathrm{mg} / \mathrm{Tag}$ & z. B. enthalten in & $\begin{array}{l}120 \mathrm{~g} \text { Petersilie } \\
175 \mathrm{~g} \text { Brokkoli, frisch } \\
115 \mathrm{~g} \text { Johannisbeeren } \\
400 \mathrm{~g} \text { Orange }\end{array}$ \\
\hline Selen & 100-200 mg/Tag & z. B. enthalten in & $\begin{array}{l}100 \mathrm{~g} \text { Hering } \\
115 \mathrm{~g} \text { Thunfisch } \\
430 \mathrm{~g} \text { Makrele } \\
135 \mathrm{~g} \text { Weizenkeime } \\
280 \mathrm{~g} \text { Weizen-VK-Brot }\end{array}$ \\
\hline Zink & 15-25 mg/Tag & z. B. enthalten in & $\begin{array}{l}100 \mathrm{~g} \text { Roggenkeime } \\
150 \mathrm{~g} \text { Weizenkleie } \\
435 \mathrm{~g} \text { fettarmer Käse } \\
400 \mathrm{~g} \text { getrocknete Linsen } \\
480 \mathrm{~g} \text { Rindfleisch }\end{array}$ \\
\hline
\end{tabular}

Quellen: Souci, Fachmann, Kraut, 7. Aufl. 2008; O. Adam: Diät + Rat bei Rheuma und Osteoporose, Walter Haedecke-Verlag, 2002 einzig Lein-, Perilla- und Rapsöl dieser Empfehlung ( $\triangleright$ Tab. 3).

Häufig wird nun Leinöl empfohlen, weil es zum einen viel Omega-3-Fettsäuren enthält und zudem ein günstiges Verhältnis der Fettsäuren zueinander aufweist. Die Überlegung, dieses Öl verstärkt den Menschen zu empfehlen, die keinen Fisch essen, ist nachvollziehbar. Die praktische Umsetzung zeigt jedoch, dass pro Tag ca. $75 \mathrm{ml}$ Leinöl eingesetzt werden müssten, um denselben Effekt zu erzielen, wie er durch den Genuss von marinen Omega-3-Fettsäuren erreicht werden kann. Diese Ölmenge ist überhaupt nicht realisierbar, weder aus geschmacklichen, noch aus koch- bzw. zubereitungstechnischen Gründen.

\section{Fischölpräparate als Alternative}

Wenn Rheumatiker Fisch essen, und besonders die Fettfische wählen, stellt die Versorgung mit der entzündungshemmenden Fettsäure EPA in der Regel kein Problem dar. Bei Betroffenen, die keinen Fisch essen, ist die herkömmliche Versorgung mit diesen wertvollen Fettsäuren in Frage gestellt, hier wird von Seiten der Wissenschaft eine dauerhafte Ergänzung mit Fischölpräparaten empfohlen. Eine dreimonatige Supplementierung über Nahrungsergänzung zur Anreicherung

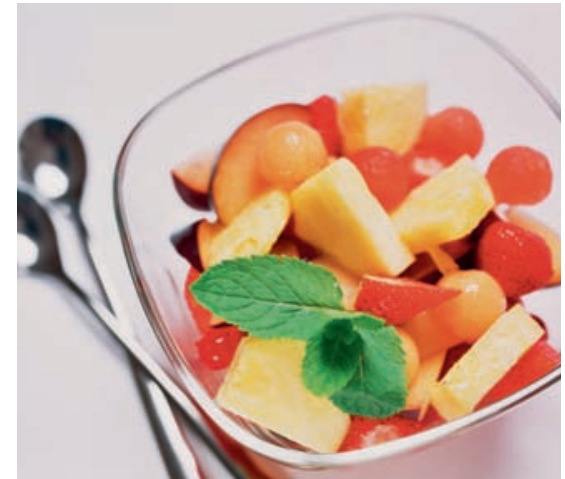

„5 am Tag“: Rheumatiker brauchen mehr Vitamin $E$ und $C$ sowie Zink und Selen. (c) DAK/Kohlbecher

der Zellmembranen scheint sehr effektiv. Hierzu ist es sinnvoll, Präparate zu wählen, die Vitamin $\mathrm{E}$ enthalten (das ist nicht bei allen angebotenen Produkten der Fall). Zudem sollte man diejenigen auswählen, die als Arzneimittel vertrieben werden. Die Vorteile bei diesen Präparaten sind die definierte Dosierung und der Beipackzettel, der über Anwendungsgebiete, Gegenanzeigen und Nebenwirkungen (z.B. erhöhte Blutungsneigung) informiert.

Übrigens: Lebertran ist nicht gleichzusetzen mit Fischöl; Rheumatiker benötigen das Öl aus dem Fisch selbst (in den Fischölkapseln enthalten) und nicht das Öl aus der Fischleber, das viel Vitamin A und D enthält.

\section{Was bringen Vitamine und Mineralstoffe?}

Die Vitamin- und Mineralstoffversorgung und -zufuhrempfehlung steht und fällt mit der täglich aufgenommenen Obst-, Gemüse- und Getreidemenge. Für Rheumatiker gelten besonders die Vitamine E und C sowie die Mineralien Selen und Zink als immunstabilisierend und sind daher in höheren Mengen empfehlenswert. Vitamin E müsste nach den Empfehlungen über Supplementierung ergänzt werden, da die Zufuhr über die dafür notwendige Nahrungsmittelmenge nicht realisiert werden kann ( $\triangleright$ Tab.4). Zahlreiche Studien haben jedoch keine ausreichenden Nachweise für die Wirksamkeit einer Supplementierung aufgezeigt. Daher bleibt diese Empfehlung vorerst offen, im Gegensatz zur Vitamin-C- 
Empfehlung. Hier kann über die gewünschten 5 Obst- und Gemüseportionen pro Tag viel aufgenommen werden.

Bei Selen und Zink ist man sich sicher, dass dies über die reguläre Ernährung schwierig ist, besonders bei Personen, die keinen Fisch essen. Hier ist Nahrungsergänzung sinnvoll. Die Datenlage der Studien ergibt jedoch noch zu wenige Erkenntnisse, um daraus noch konkretere Zufuhrempfehlungen, sei es über Nahrungsmittel oder Supplemente, für die Rheumatiker zu entwickeln.

\section{Fazit}

- Eine arachidonsäurearme Kost mit maximal 2 Fleischmahlzeiten und 2 Eigelb pro Woche bringt Vorteile. Daraus abgeleitet wird eine überwiegend lakto-vegetabile Ernährung mit vielen fettarmen Milchprodukten (unterstützt auch die Kalziumzufuhr) empfohlen.
- Eine hohe Zufuhr an Nahrungsmitteln mit vielen Omega-3-Fettsäuren (besonders EPA), wie z.B. Hering, Makrele, Lachs und Thunfisch sowie Lein-, Perilla- und Rapsöl, hemmen Entzündungsprozesse. „Nicht-Fischesser“ sollten Omega-3-Fettsäuren über Fischölpräparate ergänzen.

- Viel Obst und Gemüse („5 am Tag“) sowie oft und regelmäßig Vollkornprodukte liefern Vitamine und Mineralien, die das Immunsystem unterstützen.

\section{Online}

http://dx.doi.org/10.1055/s-0030-1255325

\section{Literatur/Quellenhinweise}

Literatur/Quellenhinweise bei der Verfasserin.

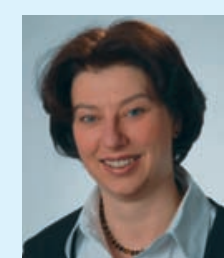

Birgit Blumenschein Havixbeckerstraße 22A 48161 Münster

Birgit Blumenschein arbeitet seit 1990 als Diätassistentin und hat Medizinpädagogik studiert. Seit 2003 selbständig engagiert sie sich im Bereich Ernährung, Ernährungsmedizin und Gesundheitsförderung. Ihre Schwerpunkte sind Individualberatungen und Gruppenschulungen, besonders zu Themen der Gastroenterologie und dem Stoffwechsel. Als Lehrbeauftragte im Studium Clinical Nutrition/Ernährungsmanagement Bsc. ist sie ebenso tätig wie als Referentin diverser Seminare und Dozentin an Fort- und Weiterbildungsinstitutionen.

blumenschein@ernaehrungstherapieblumenschein.de 